\title{
Optimization of Identifying Point Pollution Sources for the Convection-Diffusion-Reaction Equations
}

\author{
Yujing Yuan ${ }^{1, *}$ and Dong Liang ${ }^{2}$ \\ ${ }^{1}$ College of Science, Henan University of Technology, Zhengzhou, Henan 450001, China \\ 2 Department of Mathematics and Statistics, York University, Toronto, Ontario, \\ M3J 1P3, Canada
}

Received 23 April 2019; Accepted (in revised version) 5 June 2020

AMS subject classifications: 35Q93, 65M32

\begin{abstract}
In this paper, we consider the optimization problem of identifying the pollution sources of convection-diffusion-reaction equations in a groundwater process. The optimization model is subject to a convection-diffusion-reaction equation with pumping point and pollution point sources. We develop a linked optimization and simulation approach combining with the Differential Evolution (DE) optimization algorithm to identify the pumping and injection rates from the data at the observation points. Numerical experiments are taken with injections of constant rates and timedependent variable rates at source points. The problem with one pumping point and two pollution source points is also studied. Numerical results show that the proposed method is efficient. The developed optimized identification approach can be extended to high-dimensional and more complex problems.
\end{abstract}

Key words: Convection-diffusion-reaction equation, optimization of identification, pumping point, pollution source point, DE algorithm.

\section{Introduction}

A rapid increase in industrial activities is accompanied by the release of substantial quantities of pollutants. Environmental pollution has been recognized to be an important and difficult problem that needs to efficiently control the process of industrial releases, etc.

It is no doubt that identifying unknown groundwater sources is a prerequisite for the management and development decision in the water quality, which is also called as the inverse source problem of the groundwater flow. Some methodologies have been proposed for pollution source identifications, such as non-linear least-squares method [1], a geo-statistical approach [17], constrained robust least square approach [20], and so on.

*Corresponding author.

Emails: yyjyuan@haut.edu.cn (Y. J. Yuan), dliang@mathstat.yorku.ca (D. Liang) 
On the other hand, the aquifer simulation models have been combined with the optimization models to achieve groundwater management objectives for identifying unknown groundwater pollution sources $[5,6,14,16]$. In these literatures, they embeded the groundwater flow and solute transport equations into the optimization model, and the groundwater flow and solute transport equations need to be solved numerically firstly. While the numerical schemes used in papers $[5,6]$ and [14] were the Galerkin finite element and classical finite difference methods, these methods may bring nonphysical oscillation to convection dominated diffusion problems where the diffusion coefficients are small. Furthermore, the optimization algorithm of paper [5] were MINOS and NPSOL, while paper [14] used MINOS and papers [6] and [16] used Simulated Annealing (SA) and artificial neural network (ANN) respectively. Each of these optimization algorithms has advantages and limitations, authors directly used them and did not compare results there. Our constraint equations in this study are convection diffusion reaction equations, which are different with previous models. We use Differential Evolution algorithm as optimization algorithm and obtain the feasibility of our identification model.

In this paper, we study the optimization of identifying the pollution sources for a groundwater river. A linked simulation-optimization model is proposed, where the convection diffusion reaction equations are computed by the improved/upwind finite difference approach that can avoid nonphysical oscillation of numerical solutions, and the optimization is solved by the constrained Differential Evolution optimization algorithm. The advantage of the methodology is the external linking of the numerical simulation model with the optimization model. We build the objective function aimed at minimizing the weighted sum of squared deviation between observed and simulated concentrations at observation points over time, which also satisfies the constraints of the pumping and injection rates and the concentration restrictions. This process can have the physical explanation: searching the optimal rate, to minimize, in a certain range, the objective function of the simulated concentrations which satisfying the real environment at the same time. We use the constrained Differential Evolution optimization algorithm to solve the optimization problems, which provides the advantages of its global solution solving feature, simplicity, powerful search capability, compact structure, and convergence. $\mathrm{Nu}$ merical experiments are taken for constant injection rates at one to three source points respectively and for variable rates at one source point. We also consider an example with one pumping point and two pollutant source points. Numerical results show that the developed method is efficient. The method can be extended to high-dimensional and more complex problems.

This paper is organized as follows. In Section 2, we present the mathematical model of the convection diffusion reaction equations with point pollutant sources and pumping wells and give the numerical scheme for solving the convection diffusion reaction equations. In Section 3, we propose a mathematical optimization problem with constraint of numerical solution systems of PDEs. The optimization solving algorithm is described in Section 4. Numerical experiments are presented in Section 5 and some conclusions are addressed in Section 6. 


\section{Problem formalization}

\subsection{The governing equations}

Groundwater solute transport process $[7,8]$ is a very complex process. We consider an optimization problem of identifying the pollution sources from the measurement data specified at some points in a watershed. Such a model is related to the detection of pollution sources causing water contamination in some regions. With the consideration of the solute diffusion, the movement of watershed and self-purifying function of water system, the concentration of pollution $c(x, t)$ in a watershed is governed by

$$
\begin{cases}\frac{\partial c(x, t)}{\partial t}+v \frac{\partial c(x, t)}{\partial x}-D \frac{\partial^{2} c(x, t)}{\partial x^{2}}+R c(x, t)+\sum_{m=1}^{n_{p}} q_{m}^{*} c(x, t) \delta\left(x-x_{p_{m}}\right) \\ =\sum_{j=1}^{n_{s}} q_{j}(t) c^{*} \delta\left(x-x_{s_{j}}\right), & x \in[0, L], \quad t \in[0, T], \\ c(0, t)=c(L, t)=0, & t \in[0, T], \\ c(x, 0)=c_{0}(x), & x \in[0, L],\end{cases}
$$

where $v>0$ denotes the velocity of watershed movement in the direction of $x, D$ is diffusion coefficient, $R$ is a reaction coefficient describing the self-purifying function of watershed. $q_{m}^{*}$ is called as the pumping rate and $x_{p_{m}}$ is the location of pumping point, which means that the fluid flows out at this well point. $n_{p}$ is the number of pumping points. The right hand side of the equation is the source term that causes the pollution, in which $x_{s_{j}} \in[0, L], j=1, \cdots, n_{s}$ are the source locations and $q_{j}(t) \in L^{2}[0, T]$ are the injection rates satisfying $q_{j}(t)>0$ for $0 \leq t \leq T^{*}$ and $q_{j}(t) \equiv 0$ for $T^{*}<t \leq T$. This condition implies that the source does not release pollutants any more after some moment $T^{*} . n_{s}$ is the number of pollution sources. $\delta\left(x-x_{s_{j}}\right)$ is the Dirac Delta function for which will give the definition in the next subsection.

The optimization problem considered in this paper can be stated as follows. For given measurement data $c\left(x_{0_{1}}, t\right), c\left(x_{o_{2}}, t\right), 0<t \leq T$, specified at two interior observation points $0<x_{o_{1}}<x_{o_{2}}<L$, and assuming that the locations of the pollution sources and pumping points are known, we will optimally identify the pumping rates and injection rates. There is also another description that if the concentrations at $x_{o_{i}}, c\left(x_{o_{i}}, t\right)$, are expected to satisfy the standard range of environment, how can we optimally identify the strengths of the point sources and the pumping rates.

\subsection{Numerical scheme of the model}

Knowing the definition of the Dirac Delta function in the model (2.1), it has the form as

$$
\delta(x)= \begin{cases}+\infty, & x=0, \\ 0, & x \neq 0,\end{cases}
$$


satisfying the constraint condition

$$
\int_{-\infty}^{+\infty} \delta(x) d x=1
$$

It is clear that the so-called Dirac Delta "function" is not a function in conventional sense. In particular, $\delta\left(x-x_{s}\right)$ must be singular at point $x=x_{s}$. So we often adopt a sequence of functions to approximate it in the process of computation. Here we take it as

$$
\varphi_{j}(x)= \begin{cases}\frac{1}{2 \sqrt{\pi \epsilon}} \exp \left(-\frac{\left(x-x_{s_{j}}\right)^{2}}{4 \epsilon}\right), & \left|x-x_{s_{j}}\right| \leq 2 \sqrt{2 \epsilon} \\ 0, & \text { otherwise. }\end{cases}
$$

As $\epsilon \rightarrow 0$, it approximates the Dirac Delta function.

To solve the direct problem (2.1) numerically, we begin by discretizing the spatial domain by placing a grid over the domain. For convenience, we will use a uniform grid, with grid spacing $h=L / n_{x}$. The grid points are $x_{i}=i h, i=0,1, \cdots, n_{x}$. Likewise, we discretize the time domain by placing a grid on the temporal axis with grid spacing $\Delta t=T / n_{t}$, where $n_{t}>0$ is the number of total temporal steps.

Notationaly, we will define $C_{i}^{k}$ to be the approximation to the function $c(x, t)$ at the point $(i h, k \Delta t)$, and define $q_{j}^{k}$ as the approximation to $q_{j}(t)$ at $t=k \Delta t$, and $\varphi_{j}(x)$ is the approximation to Dirac Delta function as above. Here we suppose the pollution source points and pumping points are all in grid points, where $x_{s_{j}}=I_{s_{j}} h, j=1, \cdots, n_{s}, x_{p_{m}}=I_{p_{m}} h$, $m=1, \cdots, n_{p}$ and $I_{s_{j}}, I_{p_{m}}$ are integer numbers. Due to the smaller diffusion coefficients, we use the upwind finite difference to approximate the convection term $v \frac{\partial c}{\partial x}$ as the classical central difference will make numerical solution oscillate heavily.

Approximating the partial differential equation (2.1), we get the upwind numerical scheme as

$$
\begin{aligned}
& \frac{C_{i}^{k}-C_{i}^{k-1}}{\Delta t}+v \frac{C_{i}^{k}-C_{i-1}^{k}}{h}-D \frac{C_{i+1}^{k}-2 C_{i}^{k}+C_{i-1}^{k}}{h^{2}}+R C_{i}^{k}+\sum_{m=1}^{n_{p}} q_{m}^{*} C_{i}^{k} \delta_{I_{p_{m}}, i} \\
= & \sum_{j=1}^{n_{s}} q_{j}^{k} \varphi_{j}(i h), \quad i=1, \cdots, n_{x}-1, \quad k=1, \cdots, n_{t} .
\end{aligned}
$$

Remark 2.1. During the pollution procedure, as the diffusion coefficient $D$ is often much smaller than transport velocity $v$, it is very difficult to solve the convection dominated diffusion problem numerically. Furthermore, the central finite difference method and classical finite element method may introduce nonphysical oscillations into numerical solutions $[13,18]$. In paper [13], the author proposed two upwind schemes based on the generalized finite difference methods, and experiments show that numerical solutions of two-dimensional convection-dominated diffusion problems by using generalized finite difference method occur oscillation, while the upwind schemes are still stable. Paper [18] 
modified the Crank-Nicolson scheme, so that it can permit the use of a larger time step to reach a given accuracy and it is still efficient when the transport coefficients are larger than the diffusion coefficient. Thus, we adopt the upwind finite difference method instead of the central finite difference to avoid nonphysical numerical oscillation.

Then the scheme can be written as:

$$
\begin{aligned}
& \left(-v \frac{\Delta t}{h}-D \frac{\Delta t}{h^{2}}\right) C_{i-1}^{k}+\left(1+R \Delta t+v \frac{\Delta t}{h}+2 D \frac{\Delta t}{h^{2}}+\Delta t \sum_{m=1}^{n_{p}} q_{m}^{*} \delta_{I_{p_{m}}, i}\right) C_{i}^{k} \\
& +\left(-D \frac{\Delta t}{h^{2}}\right) C_{i+1}^{k}=C_{i}^{k-1}+\Delta t \sum_{j=1}^{n_{s}} q_{j}^{k} \varphi_{j}(i h) .
\end{aligned}
$$

Let

$$
\begin{aligned}
& a=-v \frac{\Delta t}{h}-D \frac{\Delta t}{h^{2}} \quad \text { and } \quad d=-D \frac{\Delta t}{h^{2}}, \\
& b_{i}= \begin{cases}1+R \Delta t+v \frac{\Delta t}{h}+2 D \frac{\Delta t}{h^{2}}+q_{m}^{*} \Delta t, & \text { if } i=I_{p_{m}}, \quad m=1, \cdots, n_{p}, \\
1+R \Delta t+v \frac{\Delta t}{h}+2 D \frac{\Delta t}{h^{2}}, & \text { else, }\end{cases}
\end{aligned}
$$

then, the coefficient matrix is

$$
\begin{aligned}
A & =\left(\begin{array}{ccccc}
b_{1} & d & & & \\
a & b_{2} & d & & \\
& \ddots & \ddots & \ddots & \\
& & a & b_{n_{x}-2} & d \\
& & b_{n_{x}-1}
\end{array}\right), \\
F^{k} & =\left(f_{1}^{k}, f_{2}^{k}, \cdots, f_{n_{x}-1}^{k}\right)^{\tau}, \\
& \text { where } f_{i}^{k}=C_{i}^{k-1}+\Delta t \sum_{j=1}^{n_{s}} q_{j}^{k} \varphi_{j}(i h) .
\end{aligned}
$$

Thus, the scheme (2.5) can be transferred into the following matrix form:

$$
A C^{k}=F^{k},
$$

where $C^{k}=\left(C_{1}^{k}, C_{2}^{k}, \cdots, C_{n_{x}-1}^{k}\right)^{\tau}, k=1, \cdots, n_{t}$, and $n_{x}, n_{t}$ are the numbers of the total spatial and temporal steps, respectively.

\section{Optimization model of identification}

The goal of the optimization of source identification model is to determine unknown pollution source characteristics, such as the discharge rates, with a constraint of numerical solution of governing equation. 
The objective is to search for a feasible set of source intensities which minimizes the deviations between the observed-concentration and simulated-concentration based on the numerical solutions of governing equations corresponding to this set of sources. This can be achieved by minimizing the weighted sum of these squared differences between observed and simulated concentrations for all spatiotemporal points.

The optimized identification model can be defined as:

$$
\underset{q_{\min } \leq q_{j}^{k}, q_{m}^{*} \leq q_{\max }}{\operatorname{Min}} \sum_{(l, k) \in Z_{c}} \omega_{l}^{k}\left[\left\langle C_{l}^{k}\right\rangle_{o b s}-C_{l}^{k}\right]^{2}
$$

subject to:

$$
\begin{aligned}
& A C^{k}=F^{k}, \\
& c_{\min } \leq C_{l}^{k} \leq c_{\max }, \quad(l, k) \in Z_{c},
\end{aligned}
$$

where the spatio-temporal combination $Z_{c}$ represents the set of the observation locations and time levels. We consider two observation points $o_{1}$ and $o_{2}$, and use $l$ to be the corresponding grid points, named as $l=\left(l_{1}, l_{2}\right)$, where $k$ takes all the temporal steps.

The weights $\omega_{l}^{k},(l, k) \in Z_{c}$, are defined as:

$$
\omega_{l}^{k}=\frac{1}{\left[\left\langle C_{l}^{k}\right\rangle_{o b s}+\eta\right]^{2}}
$$

which are intended to normalize the terms of the objective function. It is preferable to add a constant to the observation concentration to prevent small difference at low concentration to dominate the objective function. $\eta$ generally depends on the order of the concentration values. In real life problems, it may be a fraction of the difference between largest and smallest concentration values.

\section{Optimization algorithm}

We propose to use Differential Evolution (DE) algorithm [4, 9-12, 15, 19, 21] to solve the optimization problem (3.1)-(3.2b). DE algorithm was first developed in [19] for solving optimization problems over continuous domains. It has further been developed to solve the handling multi-objective problems [15], the constrained optimizations and large scale optimization problems [9-12], etc.

Most of the traditional optimization methods, such as Gradient Descent, Conjugate Gradient, Newton's method and so on, are gradient based search techniques which require auxiliary properties like differentiability and continuity of the objective function, and they also do not guarantee to obtain the global optimum. Thus, many scientists have laid emphasis on population based search technique like Evolution Algorithms and the $\mathrm{DE}$ algorithm is a special evolution algorithm. 
Comparing with other Evolution Algorithms, the advantages of using the DE algorithm for solving global designing problems are its global solution finding property, simplicity, powerful search capability, compact structure, and high accurate characteristics.

There have been many papers compared the results of DE with other optimization algorithms. For example, paper [19] lists the competence of DE with annealed version of the Nelder and Mead strategy (ANM), Adaptive Simulated Annealing (ASA), Breeder Genetic Algorithm, Evolutionary Algorithm with Soft Genetic Operators and Stochastic Differential Equations. In most instances, DE outperformed all of the above minimization approaches in terms of required number of function evaluations necessary to locate a global minimum of the test functions. In paper [22], authors list the comparing results of novel modified DE with Artificial Immune algorithm, Genetic algorithm and Particle Swarm approach. Experiments show that it has higher efficiency than the other methods in the literature on finding better feasible solutions of most constrained problems.

We present an extension of the DE algorithm to handle nonlinear constraint functions. For simplicity, we redescribe the optimized identification problem.

Let $\vec{q}=\left\{q_{j}^{k}, 1 \leq j \leq n_{s}, 0 \leq k \leq n_{t} ; q_{m}^{*}, 1 \leq m \leq n_{p}\right\} \in D$, and make it to satisfy the following optimal problem:

$$
\operatorname{Min}_{\vec{q}} f(\vec{q})=\sum_{(l, k) \in Z_{c}} \omega_{l}^{k}\left(\left\langle C_{l}^{k}\right\rangle_{o b s}-C_{l}^{k}\right)^{2}
$$

with

$$
A C^{k}=F^{k}, \quad 1 \leq k \leq n_{t},
$$

and subject to:

$$
\begin{array}{lll}
g_{l}^{k}(\vec{q})=C_{l}^{k}-c_{\max } \leq 0, & l=l_{1}, l_{2}, & 1 \leq k \leq n_{t}, \\
g_{l}^{n_{t}+k}(\vec{q})=c_{\min }-C_{l}^{k} \leq 0, & l=l_{1}, l_{2}, & 1 \leq k \leq n_{t},
\end{array}
$$

and boundary constraint: $\vec{q} \in D$, i.e., $\vec{q}_{\min } \leq \vec{q} \leq \vec{q}_{\max }$.

Definition 4.1. For above constrained optimization problem (COP) (4.1)-(4.3b), we define the function: $\phi(x): D \rightarrow R$ by

$$
\phi(\vec{q})=\sum_{(l, k) \in Z_{c}}\left(G_{l}^{k}+G_{l}^{n_{t}+k}\right),
$$

where

$$
\begin{gathered}
G_{l}^{k}(\vec{q})= \begin{cases}\frac{1}{2}, & g_{l}^{k}(\vec{q}) \leq 0, \\
\frac{1}{\left(1+\exp \left(g_{l}^{k}(\vec{q})\right)\right)}, & \text { otherwise, }\end{cases} \\
G_{l}^{n_{t}+k}(\vec{q})= \begin{cases}\frac{1}{2}, & g_{l}^{n_{t}+k}(\vec{q}) \leq 0, \\
\frac{1}{\left(1+\exp \left(g_{l}^{k}(\vec{q})\right)\right)}, & \text { otherwise. }\end{cases}
\end{gathered}
$$


Let $S=\left\{\vec{q} \mid \vec{q} \in D\right.$ and $g_{l}^{k} \leq 0$ and $\left.g_{l}^{n_{t}+k} \leq 0\right\}$. It is obvious that $\phi(\vec{q})=n_{t}$ when $\vec{q} \in S$ and $0<\phi(\vec{q})<n_{t}$ when $\vec{q} \notin S$. The more of the constraint violation number of $\vec{q}$, the less of the value of $\phi(\vec{q})$.

Definition 4.2. Suppose $\vec{q}$ and $\vec{p}$ are two different individuals from the next two generations, we define the following function:

$$
\operatorname{prior}(\vec{q}, \vec{p})= \begin{cases}1, & \phi(\vec{q})=\phi(\vec{p}) \text { and } f(\vec{q})<f(\vec{p}) \text { or } \phi(\vec{q})>\phi(\vec{p}), \\ 0, & \text { otherwise. }\end{cases}
$$

If the constraint violation number of $\vec{q}$ is less than $\vec{p}$, that is $\phi(\vec{q})>\phi(\vec{p})$, we say that $\vec{q}$ is prior to $\vec{p}$ and prior $(\vec{q}, \vec{p})=1$. The objective function value is further compared when the constraint violation numbers are equal as $\phi(\vec{q})=\phi(\vec{p})$, and the vector $\vec{q}$ with smaller objective function value $f(\vec{q})$ will prior to the other one $\vec{p}$.

Finally, we will give the description of the extended Differential Evolution algorithm:

(1) Set the control parameters. Set the number of the population $N_{p}$, mutation operator $F_{x c}$, crossover rate $C R$, the most generation $G_{\max }$ and the stop criteria.

(2) Initialize population. Set $G=0$, and define $N_{p} N$-dimension vectors, so-called individuals, which encode the candidate solutions: $\vec{q}_{i, G}=\left(q_{i, G}^{1}, \cdots, q_{i, G}^{N}\right), i=1, \cdots, N_{p}$ towards the global optimum. The initial population should better cover the entire search space as much as possible, and they are often generated by uniformly randomizing individuals within the search space constrained by the prescribed minimum and maximum parameter bounds $\vec{q}_{\min }=\left(q_{\min }^{1}, \cdots, q_{\min }^{N}\right)$ and $\vec{q}_{\max }=$ $\left(q_{\max }^{1}, \cdots, q_{\max }^{N}\right)$. For example, the initial value of the $j$ th parameter in the $i$ th individual at the generation $G$ is generated by:

$$
q_{i, G}^{j}=q_{\min }^{j}+\operatorname{rand}(\cdot) \cdot\left(q_{\max }^{j}-q_{\min }^{j}\right),
$$

where $\operatorname{rand}(\cdot)$ is a random number in $[0,1]$.

(3) Mutation operation. For vector $\vec{q}_{i, G}, i=1, \cdots, N_{p}$, a perturbed vector $\vec{v}_{i, G+1}$, called mutant vector, is generated by

$$
\vec{v}_{i, G+1}=\vec{q}_{r_{1}, G}+F_{x c} \cdot\left(\vec{q}_{r_{2}, G}-\vec{q}_{r_{3}, G}\right),
$$

where $r_{1}, r_{2}, r_{3} \in\left[1, N_{p}\right]$ are integers, they are chosen randomly, they are different from each other and different from index $i$. $F_{x c} \in[0,2]$ is a real and constant factor, which controls the amplification of the difference variation $\left(\vec{q}_{r_{2}, G}-\vec{q}_{r_{3}, G}\right)$.

Remark 4.1. It should be noted that there is not only one perturbed method, we list the other four frequently used mutation strategies implemented in the code.

$$
\begin{aligned}
& \vec{v}_{i, G+1}=\vec{q}_{b e s t, G}+F_{x c} \cdot\left(\vec{q}_{r_{1}, G}-\vec{q}_{r_{2}, G}\right) ; \\
& \vec{v}_{i, G+1}=\vec{q}_{i, G}+F_{x c} \cdot\left(\vec{q}_{b e s t, G}-\vec{q}_{i, G}\right)+F_{x c} \cdot\left(\vec{q}_{r_{1}, G}-\vec{q}_{r_{2}, G}\right) ; \\
& \vec{v}_{i, G+1}=\vec{q}_{b e s t, G}+F_{x c} \cdot\left(\vec{q}_{r_{1}, G}-\vec{q}_{r_{2}, G}\right)+F_{x c} \cdot\left(\vec{q}_{r_{3}, G}-\vec{q}_{r_{4}, G}\right) ; \\
& \vec{v}_{i, G+1}=\vec{q}_{r_{1}, G}+F_{x c} \cdot\left(\vec{q}_{r_{2}, G}-\vec{q}_{r_{3}, G}\right)+F_{x c} \cdot\left(\vec{q}_{r_{4}, G}-\vec{q}_{r_{5}, G}\right) .
\end{aligned}
$$


(4) Crossover operation. Crossover operation is applied to each pair of target vector $\vec{q}_{i, G}$ and its corresponding mutant vector $\vec{v}_{i, G+1}$ to generate a trial vector: $\vec{u}_{i, G+1}=$ $\left(u_{i, G+1}^{1}, \cdots, u_{i, G+1}^{N}\right)$. In the basic version, the $\mathrm{DE}$ algorithm employs the binomial crossover defined as bellow:

$$
u_{i, G+1}^{j}= \begin{cases}v_{i, G+1}^{j}, & \text { if } \operatorname{randb}(j) \leq C R \text { or } j=\operatorname{rnbr}(i), \\ q_{i, G^{\prime}}^{j} & \text { otherwise, }\end{cases}
$$

where $j=1, \cdots, N$, randb is a list of random number and $r a n d b(j)$ is the $j$ th number, $r n b r$ is a randomly chosen integer in $[1, N]$, the crossover rate $C R$ is a user-specified constant within the range $[0,1]$.

Remark 4.2. There exists another exponential crossover operator in addition to binomial crossover.

(5) Selection operation. After the mutation and crossover operations, the trial vector $\vec{u}_{i, G+1}$ is compared with the old vector $\vec{q}_{i, G}$. If the trial vector has an equal or better objective value, then it replaces the old vector in the next generation. This can be presented as follows:

$$
\vec{q}_{i, G+1}= \begin{cases}\vec{u}_{i, G+1}, & \text { if prior }\left(\vec{u}_{i, G+1}, \vec{q}_{i, G}\right)=1, \\ \vec{q}_{i, G}, & \text { otherwise. }\end{cases}
$$

(6) Let $G=G+1$. If $G \leq G_{\max }$ and the stop criteria is not satisfied, then turn to Step (2). Otherwise, output the optimal solution $\vec{q}_{i, G+1}$.

\section{$5 \quad$ Numerical experiments}

In this section, we will consider our optimization identification method by two kinds of examples based on whether or not the pollution source intensities depend on time $t$.

The data, used as observation data for the test of the proposed model and algorithm here, are obtained from the following relationship:

$$
\left\langle C_{l}^{k}\right\rangle_{\text {obs }}=\left\langle C_{l}^{k}\right\rangle_{\text {simul }}+\xi\left\langle C_{l}^{k}\right\rangle_{\text {simul }}(2 \operatorname{rand}(\cdot)-1) .
$$

The scheme of perturbing the numerically simulated concentration data is analogous to collecting and then testing multiple samples of contaminated water at each spatiotemporal observation location. It is assumed that observation datum obey a normal distribution. The mean of the normal distribution is the exact datum $\left\langle C_{l}^{k}\right\rangle_{\text {simul }}$, which can be obtained from the simulation with actual intensity. The standard deviation equals to some fraction $\xi$ of the exact datum, which called noise level. While $\xi=0$ means that it is error free. The $\operatorname{rand}(\cdot)$ is a random number between $[0,1]$. 


\subsection{Experiment 1}

In this subsection, we firstly give an example, that compares the improved/upwind finite difference (UFD) approach with central finite difference (CFD), to show that the central FD can bring nonphysical oscillations into numerical solutions.

We consider the following convection-dominated diffusion problem:

$$
\begin{cases}\frac{\partial c(x, t)}{\partial t}+v \frac{\partial c(x, t)}{\partial x}-D \frac{\partial^{2} c(x, t)}{\partial x^{2}}+R c(x, t)=0.5, & x \in[0,1], \quad t \in[0,1] \\ c(0, t)=c(1, t)=0, & t \in[0,1] \\ c(x, 0)=x, & x \in[0,1] .\end{cases}
$$

As shown in Fig. 1, for the convection dominated diffusion problem, we can see that the numerical solution of CFD is consistent with the numerical solution of UFD when the space step is $d x=0.01$ (a), but it will occur oscillation when the space step becomes larger (b). Since the classical central finite difference method may produce wrong solution (oscillation), we use the upwind finite difference method to solve the convection-dominated diffusion equations which are the constraint equations of the optimization model.

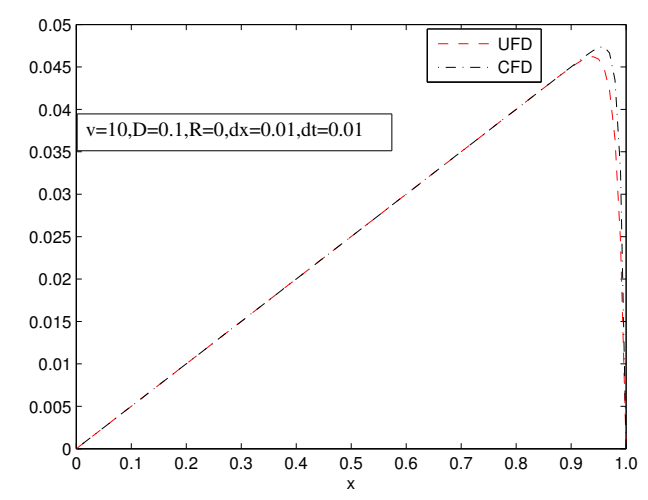

(a)

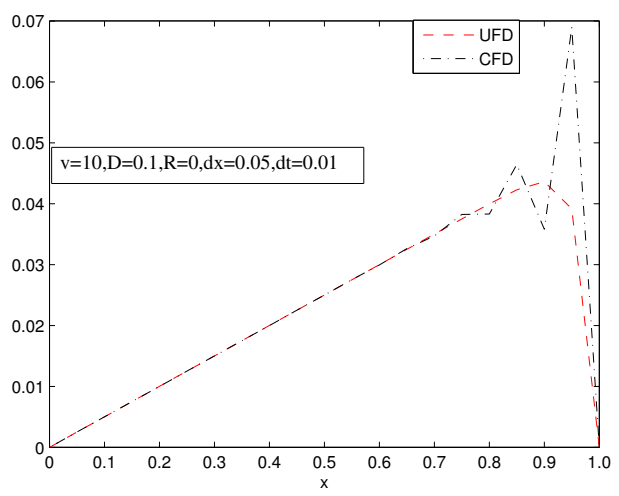

(b)

Figure 1: Numerical solutions by using UFD and CFD when $v=10.0, D=0.1$ and $R=0$ with steps (a): $d x=0.01$, $d t=0.01$ and (b): $d x=0.05, d t=0.01$.

\subsection{Experment 2}

In this example, we suppose that there is no pumping point, the pollution flow is in a portion of the river of length $L=1$ during a period $T=1.2$ and with $T^{*}=1.0$. The diffusion coefficient is $D=0.05$, the velocity of the watershed is $v=1.0$ and the selfpurifying coefficient is $R=0.125$. The initial condition that we take here is $c_{0}(x)=0$.

We take $h=0.01, \Delta t=0.01$. Suppose each source's injection rate is constant during all the active time $t$. In this example, three cases are studied. 
Table 1: Identified results of injection rates with source location at $s_{1}=0.4$, observation locations at $o_{1}=0.1$ and $o_{2}=0.6$, and with different noise $\xi$.

\begin{tabular}{||c|c|c|c|c||}
\hline \multirow{2}{*}{} & \multirow{2}{*}{ actual rate } & \multicolumn{3}{|c|}{ optimized identified rate } \\
\cline { 3 - 5 } & & $\xi=0$ & $\xi=0.005$ & $\xi=0.01$ \\
\hline$q_{1}$ & 0.3 & 0.3 & 0.2999735 & 0.2998348 \\
\hline
\end{tabular}

Table 2: Identified results of injection rates with source location at $s_{1}=0.4$, observation locations at $o_{1}=0.1$ and $o_{2}=0.6$, and with different noise $\xi$.

\begin{tabular}{||c|c|c|c|c||}
\hline \multirow{2}{*}{} & \multirow{2}{*}{ actual rate } & \multicolumn{3}{|c||}{ optimized identified rate } \\
\cline { 3 - 5 } & & $\xi=0$ & $\xi=0.005$ & $\xi=0.01$ \\
\hline$q_{1}$ & 0.25 & 0.2499096 & 0.2499085 & 0.2498493 \\
\hline
\end{tabular}

Table 3: Numerical results of injection rates with source locations at $s_{1}=0.2$ and $s_{2}=0.4$, the observation locations at $o_{1}=0.1$ and $o_{2}=0.6$, and with different noise $\xi$.

\begin{tabular}{||c|c|c|c|c||}
\hline \multirow{2}{*}{ actual rate } & \multicolumn{3}{|c||}{ optimized identified rate } \\
\cline { 3 - 5 } & & $\xi=0$ & $\xi=0.005$ & $\xi=0.01$ \\
\hline$q_{1}$ & 0.2 & 0.2 & 0.1999599 & 0.1995368 \\
\hline$q_{2}$ & 0.3 & 0.3 & 0.2999852 & 0.3000880 \\
\hline
\end{tabular}

Table 4: Numerical results of injection rates with source locations at $s_{1}=0.2$ and $s_{2}=0.4$, the observation locations at $o_{1}=0.1$ and $o_{2}=0.6$, and with different noise $\xi$.

\begin{tabular}{||c|c|c|c|c||}
\hline \multirow{2}{*}{} & \multirow{2}{*}{ actual rate } & \multicolumn{3}{|c||}{ optimized identified rate } \\
\cline { 3 - 5 } & & $\xi=0$ & $\xi=0.005$ & $\xi=0.01$ \\
\hline$q_{1}$ & 12.0 & 11.99995 & 11.99934 & 12.01011 \\
\hline$q_{2}$ & 18.0 & 17.99947 & 17.98531 & 17.98590 \\
\hline
\end{tabular}

In the first case, we consider the simplest situation where there is only one pollution source point at $s_{1}=0.4$, the observation points are situated at $o_{1}=0.1$ and $o_{2}=0.6$. Performance evaluations are done for error free and with noise level of $\xi=0.005$ and $\xi=0.01$ in observation data. The numerical results obtained by solving the proposed optimized source identification model are presented in Tables 1-2. Here, we use two different actual injection rates to check the feasibility of the method.

The second case considers a problem with two pollution sources located at $s_{1}=0.2$ and $s_{2}=0.4$. The locations of the observation points are same as the first case. Numerical evaluations are done for error free and with noise level of $\xi=0.005$ and $\xi=0.01$ in observation data. The numerical results obtained by solving the proposed optimized source identification model are presented in Tables 3-4, which also use two sets of different actual injection rates to check the feasibility.

In the third case, we consider three pollution source points at $s_{1}=0.2, s_{2}=0.4$ and $s_{3}=0.6$, and two observation points at $o_{1}=0.1$ and $o_{2}=0.8$. Numerical computations are done like the former two cases. The results for using two different sets of actual injection 
Table 5: Results of injection rates with source locations at $s_{1}=0.2, s_{2}=0.4$ and $s_{3}=0.6$, the observations at $o_{1}=0.1$ and $o_{2}=0.8$, and with different noise $\xi$.

\begin{tabular}{||c|c|c|c|c||}
\hline \multirow{2}{*}{} & \multirow{2}{*}{ actual rate } & \multicolumn{3}{|c||}{ optimized identified rate } \\
\cline { 3 - 5 } & & $\xi=0$ & $\xi=0.005$ & $\xi=0.01$ \\
\hline$q_{1}$ & 0.2 & 0.2 & 0.2000546 & 0.2001756 \\
\hline$q_{2}$ & 0.3 & 0.3000001 & 0.3000014 & 0.2983591 \\
\hline$q_{3}$ & 0.4 & 0.3999996 & 0.4003360 & 0.4011268 \\
\hline
\end{tabular}

Table 6: Results of injection rates with source locations at $s_{1}=0.2, s_{2}=0.4$ and $s_{3}=0.6$, the observation locations at $o_{1}=0.1$ and $o_{2}=0.8$, and with different noise $\xi$.

\begin{tabular}{||c|c|c|c|c||}
\hline \multirow{2}{*}{} & \multirow{2}{*}{ actual rate } & \multicolumn{3}{|c||}{ optimized identified rate } \\
\cline { 3 - 5 } & & $\xi=0$ & $\xi=0.005$ & $\xi=0.01$ \\
\hline$q_{1}$ & 21.0 & 20.99987 & 20.99662 & 20.96585 \\
\hline$q_{2}$ & 15.0 & 14.99824 & 14.98989 & 15.01102 \\
\hline$q_{3}$ & 4.0 & 4.000161 & 4.000209 & 4.011969 \\
\hline
\end{tabular}

rates are given in Tables 5-6.

From Tables 1-6, we can see clearly that the numerical optimized identified rates are in a great agreement with actual values. They are almost equal to actual rates, when concentration measurement data have small error. They have errors when some bigger noises are used in observed data.

\subsection{Experment 3}

For this example, physical parameters of the problem are the same with Experiment 2, while the pumping points are also involved.

We set the pumping point at $p_{1}=0.5$, the pollution sources at $s_{1}=0.2$ and $s_{2}=0.6$, and the observation points at $o_{1}=0.1$ and $o_{2}=0.8$. Tables 7-8 give the numerical results using different noise levels in observation concentration measurement data. The pumping rate and source injection rates are efficiently identified with different noise errors. Meanwhile, while the source injection rates can be simulated more exact with noise involved, the simulated pumping rate has a reasonable identification comparing with the actual value.

While the weights in the objective function are important and relative with the simulated concentration using the actual source fluxes, we give the comparisons of performed evaluations with different weights. The numerical results are shown with different $\eta$ in Tables 9-10. We can see that the good simulation results are based on a certain order of magnitude of $\eta$. 
Table 7: Numerical Results of pumping rate and injection rate with source location ats $p_{1}=0.5, s_{1}=0.2$, and $s_{2}=0.6$, the observation locations at $o_{1}=0.1$ and $o_{2}=0.8$.

\begin{tabular}{||c|c|c|c|c||}
\hline \multirow{2}{*}{} & \multirow{2}{*}{ actual rate } & \multicolumn{3}{|c||}{ optimized identified rate } \\
\cline { 3 - 5 } & & $\xi=0$ & $\xi=0.005$ & $\xi=0.01$ \\
\hline$q_{1}^{*}$ & 2.0 & 1.999949 & 2.022508 & 2.0252894 \\
\hline$q_{1}$ & 0.2 & 0.2 & 0.1999453 & 0.2000317 \\
\hline$q_{2}$ & 0.4 & 0.3999996 & 0.3999298 & 0.4003350 \\
\hline
\end{tabular}

Table 8: Numerical results of pumping rate and injection rate with source locations at $p_{1}=0.5, s_{1}=0.2$ and $s_{2}=0.6$, the observation locations at $o_{1}=0.1$ and $o_{2}=0.8$.

\begin{tabular}{||c|c|c|c|c||}
\hline \multirow{2}{*}{} & \multirow{2}{*}{ actual rate } & \multicolumn{3}{|c||}{ optimized identified rate } \\
\cline { 3 - 5 } & & $\xi=0$ & $\xi=0.005$ & $\xi=0.01$ \\
\hline$q_{1}^{*}$ & 20.0 & 19.99995 & 20.11325 & 20.50563 \\
\hline$q_{1}$ & 11.0 & 11.00000 & 11.00486 & 11.00397 \\
\hline$q_{2}$ & 17.0 & 17.00000 & 16.99973 & 17.01842 \\
\hline
\end{tabular}

Table 9: Comparison for results of pumping rate and injection rates with noise level $\xi=0.005$ and with different $\eta$.

\begin{tabular}{||c|c|c|c|c||}
\hline \multirow{2}{*}{} & \multirow{2}{*}{ actual rate } & \multicolumn{3}{|c||}{ optimized identified rate } \\
\cline { 3 - 5 } & & $\eta=10^{-7}$ & $\eta=10^{-6}$ & $\eta=10^{-5}$ \\
\hline$q_{1}^{*}$ & 2.0 & 2.099947 & 2.022508 & 2.100008 \\
\hline$q_{1}$ & 0.2 & 0.1999494 & 0.1999453 & 0.1999785 \\
\hline$q_{2}$ & 0.4 & 0.4001339 & 0.3999298 & 0.4002180 \\
\hline \multicolumn{2}{|c|}{ minimum objective } & $0.1454428 \mathrm{E}-02$ & $0.1380294 \mathrm{E}-02$ & $0.1421481 \mathrm{E}-02$ \\
\hline
\end{tabular}

Table 10: Comparison for results of pumping rate and injection rate with noise level $\xi=0.005$ and different $\eta$.

\begin{tabular}{||c|c|c|c|c||}
\hline \multirow{2}{*}{} & \multirow{2}{*}{ actual rate } & \multicolumn{3}{|c||}{ optimized identified rate } \\
\cline { 3 - 5 } & & $\eta=10^{-5}$ & $\eta=10^{-4}$ & $\eta=10^{-3}$ \\
\hline$q_{1}^{*}$ & 20.0 & 20.16759 & 20.11325 & 20.01635 \\
\hline$q_{1}$ & 11.0 & 10.99613 & 11.00486 & 10.99395 \\
\hline$q_{2}$ & 17.0 & 17.00539 & 16.99973 & 16.99404 \\
\hline \multicolumn{2}{|c|}{ minimum objective } & $0.1440363 \mathrm{E}-02$ & $0.1412140 \mathrm{E}-02$ & $0.1438028 \mathrm{E}-02$ \\
\hline
\end{tabular}

\subsection{Experment 4}

We consider the pollution process that the source intensity is time dependent as

$$
q(t)=\sum_{k=1}^{4} \alpha_{k} e^{-\beta_{k}\left(t-t_{k}\right)^{2}}, \quad 0 \leq t<1,
$$

at different time intervals, where the configuration coefficients are $\alpha_{1}=12, \alpha_{2}=17, \alpha_{3}=10$, $\alpha_{4}=5, \beta_{1}=120, \beta_{2}=80, \beta_{3}=180, \beta_{4}=120, t_{1}=0.2, t_{2}=0.5, t_{3}=0.7, t_{4}=0.9$. The problem 


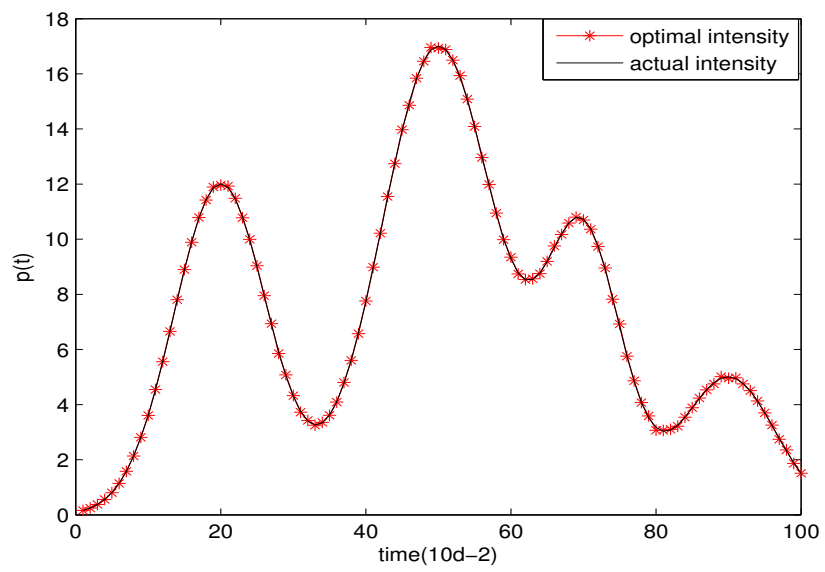

Figure 2: Results of injection rate with source location at $s=0.5$, the observation locations at $o_{1}=0.4$ and $\mathrm{o}_{2}=0.6$, and with noise level $\xi=0$.

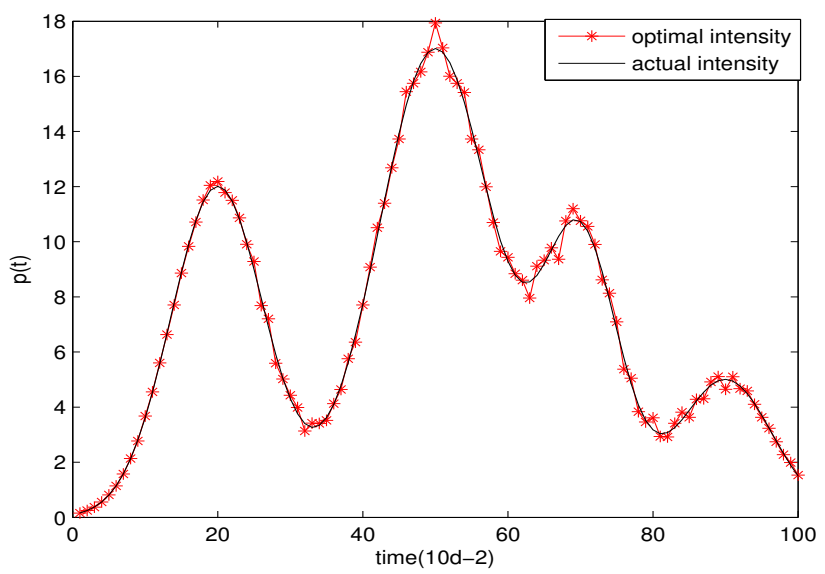

Figure 3: Results of injection rate with source location at $s=0.5$, the observation location at $o_{1}=0.4$ and $o_{2}=0.6$, and with noise level $\xi=0.005$.

is with $D=0.5, v=3.5$ and $R=0.145$, and $L=1, T=1.2, T^{*}=1.0$. The initial condition that we take here is $c_{0}(x)=0$.

The location of the pollution source point is at $s=0.5$, and the observation points are at $o_{1}=0.4$ and $o_{2}=0.6$. In the numerical computation, we take the space and time step as $d x=d t=0.01$. The comparisons of the numerical injection rates that obtained by solving the optimized identification model and actual injection rates are shown in Figs. 2-4. The results demonstrate that the simulated values are in an excellent agreement with actual values. 


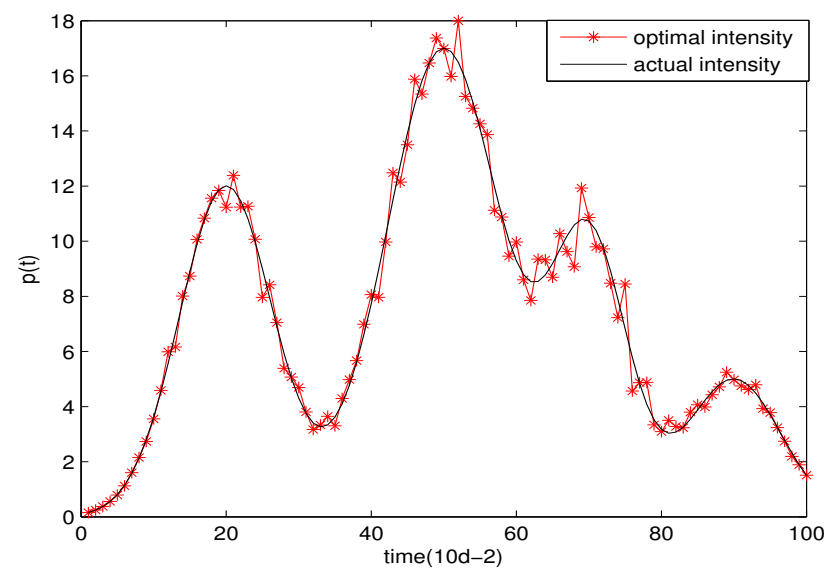

Figure 4: Results of injection rate with source location at $s=0.5$, the observation locations at $o_{1}=0.4$ and $o_{2}=0.6$, and with noise level $\xi=0.01$.

\section{Conclusions}

In this paper, we considered an optimization problem of identifying the point source detection in a watershed. The point source intensities were studied based on the observed values at observation points. We developed a linked simulation-optimization model with the DE optimization algorithm for the problems.

We first showed that the improved/upwind finite difference scheme is better for convection dominated diffusion problem than the central finite difference scheme. We then took the source injection rates as constants in two examples of optimization problems of identifying the point source detection, while the second one with a pumping point. We further tested the examples with one pollution point and time dependent rate $q(t)$ as the source intensity. We compared the simulation results with actual values for different observation data by giving different perturbation. The comparisons of different weights in the objective function were also studied.

Because of the advantages of the DE algorithm, the linked optimized identification model can be applied to solve other more sophisticated problems in groundwater. We can also adopt other more efficient numerical algorithm to solve two-dimensional problems as a future work.

\section{Acknowledgements}

The authors would like to thank the referees for their valuable comments that have helped to improve the paper. This work was supported by Natural Sciences and Engineering Research Council of Canada, and by the Doctor Research Foundation for Advanced Talents (No. 2018BS026) of Henan University of Technology. 


\section{References}

[1] S. AlAPATI AND Z. J. KABALA, Recovering the release history of a groundwater contaminant using a non-linear least-squares method, Hydrol. Process., 14 (2000), pp. 1003-1016.

[2] M. M. ARAL, J. B. GUAN AND M. L. MASLIA, Identification of contaminant source location and history in aquifers, J. Hydrol. Eng., 6(3) (2001), pp. 225-234.

[3] M. T. AYVAZ, A linked simulation-optimization model for solving the unknown groundwater pollution source identification problems, J. Contam. Hydrol., 117 (2010), pp. 46-59.

[4] S. DAS AND P. N. SUGANTHAN, Differential evolution: a survey of the state-of-the-art, IEEE Transaction on Evolutionary Computation, 15(1) (2011), pp. 4-31.

[5] B. DATTA, D. CHAKRABARTY AND A. DHAR, Identification of unknown groundwater pollution sources using classical optimization with linked simulation, J. Hydro-Environ. Res., 5 (2011), pp. 25-36.

[6] B. DATTA, O. PRAKASH, S. CAMPBELl AND G. EsCALADA, Efficient identification of unknown groundwater pollution sources using linked simulation-optimization incorporating monitoring location impact factor and frequency factor, Water Resour. Manage., 27 (2013), pp. 4959-4976.

[7] J. DOUGLAS AND JR. J. E. ROBERTS, Numerical methods for a model for compressible miscible displacement in porous media, Math. Comput., 41(164) (1983), pp. 441-459.

[8] C. DU AND D. LIANG, An efficient S-DDM iterative approach for compressible contamination fluid flows in porous media, J. Comput. Phys., 229 (2010), pp. 4501-4521.

[9] W. Y. GONG, Z. H. CAI AND D. W. LIANG, Engineering optimization by means of an improved constrained differential evolution, Comput. Methods Appl. Mech. Eng., 268 (2014), pp. 884904.

[10] Y. C. HE AND X. Z. WANG, Solution of hard constrained optimization problem based on modified Differential Evolution algorithm, Comput. Eng., 34(13) (2008), pp. 193-194.

[11] S. KUKKONEN AND J. LAMPINEN, Constrained real-parameter optimization with generalized differential evolution, IEEE Congress on Evolutionary Computation, (2006), pp. 207-214.

[12] J. LAMPINEN, A constraint handling approach for the differential evolution algorithm, IEEE Computer Society, (2002), pp. 1468-1473.

[13] D. LIANG, A kind of upwind scheme for convection-diffusion equations, Numer. Math. Sincia, 13 (1991), pp. 133-141.

[14] P. S. MAHAR AND B. DATTA, Optimal monitoring network and ground-water-pollution source identification, J. Water Resour. Plan. Manage., 123(4) (1997), pp. 199-207.

[15] S. SHARMA AND G. P. RANGAIAH, An improved multi-objective differential evolution with a termination criterion for optimizing chemical processes, Comput. Chem. Eng., 56 (2013), pp. 155-173.

[16] R. M. SINGH, B. DATTA AND A. JAIN, Identification of unknown groundwater pollution sources using artificial neural networks, J. Water Resour. Plan. Manage., 130(6) (2004), pp. 506-514.

[17] M. F. SNODGRASS AND P. K. KITANIDIS, A geostatistical approach to contaminant source identification, Water Resour. Res., 33(4) (1997), pp. 537-546.

[18] L. J. SONG AND Y. J. WU, A modified Crank-Nicolson scheme with incremental unknowns for convection dominated diffusion equations, Appl. Math. Comput., 215 (2010), pp. 3293-3301.

[19] R. STORN AND K. PRICE, Differential evolution-a simple and efficient heuristic for global optimization over continuous spaces, J. Glob. Optim., 11 (1997), pp. 341-359.

[20] A. Y. Sun, S. L. PAinter AND G. W. WitTmeyer, A constrained robust least squares approach for contaminant release history identification, Water Resour. Res., 42 (2006), W04414, doi:10.1029/2005ER004312. 
[21] J. T. TSAI, Improved Differential Evolution algorithm for nonlinear programming and engineering design problems, Neurocomputing, 148 (2015), pp. 628-640.

[22] D. X. ZOU, H. K. LIU, L. Q. GAO AND S. LI, A novel modified differential evolution algorithm for constrained optimization problems, Comput. Math. Appl., 61 (2011), pp. 1608-1623. 